\title{
EXPONENTIAL TYPE LOCALLY GENERALIZED STRICTLY DOUBLE DIAGONALLY TENSORS AND EIGENVALUE LOCALIZATION
}

\author{
JiANZHOU LIU AND LiANG XiONG
}

\begin{abstract}
In this paper, we introduce exponential type locally generalized strictly double diagonally dominant tensors. This concept extends the concept of strictly diagonally dominant tensors. It is shown that exponential type locally generalized strictly double diagonally dominant tensors must be $H$-tensors. Furthermore, as applications of exponential type locally generalized strictly double diagonally dominant tensors, we present some new eigenvalue localization sets and checkable sufficient condition for the positive definiteness of even-order real symmetric tensors. Appropriate numerical examples are proposed to illustrate that our new tensors eigenvalue localization sets are more precise than some existing sets in some cases.
\end{abstract}

Mathematics subject classification (2010): 15A18, 15A69, 15A21.

Keywords and phrases: Locally generalized strictly double diagonally tensor, $H$-tensor, tensor eigenvalue localization, positive definiteness.

\section{REFERENCES}

[1] QI, LiQUN, Eigenvalues of a real supersymmetric tensor, J. Symbolic Comput., 40, 1302-1324, 2005.

[2] Lim, LH., Singular values and eigenvalues of tensors: a variational approach, In: CAMSAP05: Proceeding of the IEEE International Workshop on Computational Advances in Multi-Sensor Adaptive Processing, 129-132, 2005.

[3] VARGA, RS., Gersgorin and his circles, Berlin,Springer-Verlag, 2004.

[4] YANG, YN. AND YANG, QZ., Further results for Perron-Frobenius theorem for nonnegative tensors, SIAM J. Matrix Anal. Appl., 31, 2517-2530, 2010.

[5] YANG, QZ. AND YANG, YN., Further results for Perron-Frobenius theorem for nonnegative tensors II, SIAM J. Matrix Anal. Appl., 32, 1236-1250, 2011.

[6] Li, CQ., LI, YT. AND KONG, X., New eigenvalue inclusion sets for tensors, Numer. Linear Algebra Appl., 21, 39-50, 2014.

[7] Li, CQ., WANG,F., ZHAO, JX. ET AL., Criterions for the positive definiteness of real supersymmetric tensors, J. Comput. Appl. Math., 255, 1-14, 2014.

[8] SHAo,JY., A general product of tensors with applications, Linear Algebra Appl., 439, 2350-2366, 2013.

[9] ZhAng, LP., QI, LQ. And Zhou, GL., M-tensors and some applications, SIAM J. Matrix Anal. Appl., 35, 437-452, 2014.

[10] ZhaO,RJ., GAO, L., LIU, QL. ET AL., Criterions for identifying $H$-tensors, Front. Math. China., 11 , 661-678, 2016.

[11] Ding, WY., QI,LQ. AND WEI,YM., M-tensors and nonsingular M-tensors, Linear Algebra Appl., 439, 3264-3278, 2013.

[12] Lathauwer, LD, Moor, BD. And Vandewalle, J. On the best rank-1 and rank- $(R 1, R 2, \ldots$ ,RN) approximation of higher-order tensors, SIAM J. Matrix Anal. Appl., 21, 1324-1342, 2000.

[13] QI, LQ., SUn, WY. AND WANG, YJ., Numerical multilinear algebra and its applications, Front. Math. China., 2, 501-526, 2007.

[14] NG, M., QI, LQ. AND ZHOU, GL., Finding the largest eigenvalue of a nonnegative tensor, SIAM J. Matrix Anal. Appl., 31, 1090-1099, 2009. 
[15] NI, Q., QI, LQ. AND WANG, F., An eigenvalue method for the positive definiteness identification problem, IEEE Trans. Automat. Control., 53, 1096-1107, 2008.

[16] Chang, KC., Pearson, KJ. And Zhang, T., Perron-Frobenius theorem for nonnegative tensors, Commun. Math. Sci., 6, 507-520, 2008.

[17] Chang, KC., Pearson, KJ. And Zhang, T., Primitivity, the Convergence of the NQZ Method, and the Largest Eigenvalue for Nonnegative Tensors, SIAM J. Matrix Anal. Appl., 32, 806-819, 2011.

[18] Friedland, S., Gaubert, S. And Han, L., Perron-Frobenius theorem for nonnegative multilinear forms and extensions, Linear Algebra Appl., 438, 738-749, 2013.

[19] LiU, Y J., ZHOU, GL. AND IBRAHIM, NF., An always convergent algorithm for the largest eigenvalue of an irreducible nonnegative tensor, J.Comput. Appl. Math., 235, 286-292, 2010.

[20] HuAng, ZG., WANG, LG., XU, Z. ET AL., A new S-type eigenvalue inclusion set for tensors and its applications, J. Inequal.Appl., 254, 1-19, 2016.

[21] LI, CQ., JIAO, AQ. AND LI, Y.T., An S-type eigenvalue localization set for tensors, Linear Algebra Appl., 493, 469-483, 2016.

[22] LI, CQ. AND LI,Y.T., MB-tensors and MB 0 -tensors, Linear Algebra Appl., 484, 141-153, 2014.

[23] LI,Y.T. ANd LI, CQ., Double B-tensors and quasi-double B-tensors, Linear Algebra Appl., 466, 343-356, 2015.

[24] QI, L. AND Song, Y., An even order symmetric B-tensor is positive definite, Linear Algebra Appl., 457, 303-312, 2014.

[25] QI,LQ., Symmetric nonnegative tensors and copositive tensors, Linear Algebra Appl., 457, 228-238, 2013.

[26] QI,LQ., Hankel Tensors: Associated Hankel Matrices and Vandermonde Decomposition, Commun. Math. Sci., 13, 1, 2014.

[27] QI,LQ. AND Y. SONG,L., Infinite and finite dimensional Hilbert tensors, Linear Algebra Appl., 451, $1-14,2014$.

[28] Yuan,PZ. And You, LH., Some remarks on $P, P_{0}, B$ and $B_{0}$ tensors, Linear Algebra Appl., 459, 511-521, 2014.

[29] Song, Y. And QI, L., Properties of Some Classes of Structured Tensors, J. Optimiz. Theory. App., $165,3,854-873,2015$.

[30] Kannan, MR., Shaked-Monderer, N. And Berman, A., Some properties of strong $H$-tensors and general $H$-tensors, Linear Algebra Appl., 476, 42-55, 2015.

[31] LI, YT., LIU, QL. AND QI, LQ., Progranmmable criteria for strong $H$-tensors, Numer. Algorithms., 74, 1, 1-23, 2016.

[32] Wang, XZ. AND WeI, YM., $H$-tensors and nonsingular $H$-tensors, Front. Math. China., 11, 3, 557-575, 2016.

[33] Hu, SL., HuAng,ZH. AND QI,LQ., Strictly nonnegative tensors and nonnegative tensor partition, Sci. China Math., 57, 1, 181-195, 2014.

[34] YANG, YUNING AND YANG, QINGZHI, On some properties of nonnegative weakly irreducible tensors, Mathematics., 74, 74, 701-711, 2012. 